\title{
Historia de la Anatomía Comparada de los Elementos Fonatorios y Articulatorios
}

\author{
History of Comparative Anatomy of the Phonatory and Articulatory Elements
}

\author{
Jorge Eduardo Duque Parra*,**; Johanna Alexandra Barco Cano* \& Juan David Sánchez Ramírez ${ }^{* * *}(\dagger$ In memoriam)
}

DUQUE, P. J. E.; BARCO, C. J. A. \& SÁNCHEZ, R. J. D. História de la anatomía comparada de los elementos fonatorios y articulatorios. Int. J. Morphol., 32(4):1337-1340, 2014.

RESUMEN: El objetivo del presente estudio fue ordenar a lo largo del tiempo, desde épocas remotas hasta nuestro tiempo, las ideas imperantes sobre los órganos fonatorios y articulatorios con base en la anatomía funcional y comparada. Se revisaron variadas fuentes bibliográficas sobre los órganos fonatorios y articulatorios y se ordenaron en el tiempo en relación a los conceptos sobre la constitución y funcionalidad de tales órganos, comparando dichas ideas y conceptos. Históricamente se muestra que con el avance de la investigación y con el desarrollo de nuevas ideas y comprobaciones empíricas se llegó a una mejor comprensión de los elementos fonatorios y articulatorios.

PALABRAS CLAVE: Historia; Fonación; Articulación; Anatomía.

\section{INTRODUCCIÓN}

El estudio de la anatomía comparada se pierde en el tiempo, posiblemente está ligado a los acercamientos de los primeros representantes de nuestra especie, quienes trataron de comprender su propia morfología comparándola con la de aquellos animales que cazaban en su lucha por la subsistencia. Esa búsqueda ancestral del conocimiento se pierde, por lo tanto, mas allá de la historia escrita. Por ejemplo, ahora se sabe que la laringe, como parte de nuestra anatomía para la fonación, es la estructura filogenéticamente más antigua, la cual apareció en los peces pulmonados hace unos 400 millones de años, aunque las primeras vocalizaciones se dieron en los anuros 250 millones de años atrás (Simonyan \& Horwitz, 2011). Pero la historia conocida más cercana del estudio de la anatomía comparada se remonta hasta Aristóteles (384-322 a.n.e) de Estagira, quien basaba sus investigaciones de la información que obtenía de animales (von Staden, 1992; Prives et al., 1984) y humanos (Lyons \& Petrucelli, 1978; Sanchez Guisande, 1945), aunque nunca llegó a disecar cadáveres humanos (García Ballester, 1973); sí examinó fetos (García Ballester; Sanchez Guisande; Prives et al.).

Aristóteles se basó en el experimentalismo como método para su conocimiento (Lyons \& Petrucelli; García
Ballester), y realizó escritos de anatomía sobre organismos vertebrados e invertebrados, razón por la cual se le conoce como el fundador de la anatomía comparada (Lyons \& Petrucelli; Gross, 1999, Prives et al.) puesto que estudió las semejanzas y diferencias de sus partes (García Ballester). Aristóteles indicaba que "tenemos que describir primero las funciones comunes; es decir, comunes a todo el reino animal o a ciertos grupos grandes o a miembros de una especie" (Coleman, 2002).

Los elementos relacionados con el aparato fonador y articulador incluyen la lengua, la laringe y los nervios que inervan la musculatura correspondiente, entre otros. La laringe, cuya musculatura estriada esquelética intrínseca es suplida por el nervio laríngeo recurrente (Aquino et al., 2001), fue estudiada a lo largo de la historia por varios autores. Se sabe, por ejemplo, que poco después de Aristóteles, Erasistratus (c. 310-250 a.n.e) realizó estudios de anatomía comparada entre distintas especies para tratar de deducir la relación entre estructura y función de esta partes anatómicas. Él describió la función de bloqueo que realiza el cartílago epiglótico al paso del aire durante la deglución (Lyons \& Petrucelli; Finger, 1994) para que no ingrese alimento al interior laríngeo (López Piñero, 2007). Esta idea revolucio-

\footnotetext{
* Departamento de Ciencias Básicas. Programa de Medicina. Universidad de Caldas, Manizales, Colombia.

** Departamento de Ciencias Básicas Biológicas. Universidad Autónoma de Manizales, Manizales, Colombia.

**** Programa de Medicina Veterinaria. Universidad de Caldas, Manizales, Colombia.
} 
nó el pensamiento de la época en torno a la deglución y a la respiración, pues se creía por ese entonces que el alimentos, entre ellos los líquidos, podían ingresar tanto hacia el estómago (por vía faringo esofágica) como hacia los pulmones (por vía faringo laríngea) (Repici, 1990).

Por la época de Erasistratus la disección de cadáveres humanos era muy restringida, lo que le obligó a realizar trabajos de anatomía comparada en los que describió similitudes entre las especies animales con el fin de deducir la relación estructura-función (Finger). Siglos después, Aurelius Celsus (25-50 a.n.e) le dio capital importancia a la lengua para la fonación, pues creía que era esta estructura oral y no la laringe la responsable de la mayoría de los trastornos del habla (Finger). Fue Soranu de Ephesus (siglo II) quién distinguió entre la parálisis de la lengua y otras enfermedades que podían causar falla en la articulación y pérdida del habla (Finger), con lo que dio a entender que la lengua no era el único órgano implicado en la producción de la voz. Años después se supo que la laringe estaba asociada funcionalmente porque anatómicamente hay vínculos mucoconectivos, los pliegues gloso-epiglóticos de la lengua (Meng et al., 2008), y que era el órgano articulatorio de la palabra. Actualmente se sabe que es el cerebro el órgano gestor de la palabra, concretamente el giro frontal inferior del hemisferio izquierdo de la mayoría de las personas (Embick et al., 2000), donde sus eferencias cortico-fugales se proyectan al tronco encefálico y de allí por el nervio accesorio, pero se continúan por el nervio vago para finalizar en la musculatura intrínseca laríngea mediante ramas terminales del nervio laríngeo recurrente (ramo del nervio vago) (Singh \& Husain, 2011).

Por el mismo tiempo de Soranu, en el siglo II, Galeno (130-200) aportó al conocimiento de la anatomía comparada disecando numerosos animales (von Staden), y describió en gran detalle las ramas del nervio vago. Sus aportes se reconocen en Omnia opera, publicada en 1541 en Venecia, donde se le muestra realizando una brillante demostración cortando el nervio laríngeo recurrente en un cerdo (Gross), acentuando su importancia anatómica y funcional, pues al hacer la vivisección de dicho animal notó que el pinzamiento del nervio abolía la generación de sus ruidos (Lyons \& Petrucelli; Gross). Además, Galeno fue el primer anatomista en describir el nervio laríngeo recurrente como una rama del nervio vago (Ardito et al., 2007; Baldoncini et al., 2011), aunque se considera que este nervio fue descrito por Marino alrededor del año 100, pero su nombre fue acuñado por Domenico de Marchetti de Padua (1626-1688) (Velásquez, 2008).

Posterior a Galeno y durante la edad media no hubo ningún aporte al conocimiento sobre la relación entre cere- bro y lenguaje. Incluso, Avicenna no da referencias respecto del déficit en la fonación y el habla (Finger). Esto se debió a que en ese tiempo las doctrinas clásicas no fueron cuestionadas, de tal manera que lo que se enseñaba era basado en el peso de la tradición, con el soporte de la filosofía y la religión más que en los hechos empíricos. Se creía incluso que las afasias eran producto de la pérdida de la memoria de las palabras, las cuales se alojaban en el cuarto ventrículo (Finger) del tronco encefálico.

Unos trece siglos después de Galeno, Jacobo Berengario da Carpi (1469-1530) describió anatómicamente los cartílagos aritenoides (Entralgo, 1982; Pérez Ibáñez, 2008) sobre los cuales se fijan músculos necesarios para la fonación, como los tiroaritenoideos, inervados por el nervio laríngeo recurrente (Simonyan). Por esa misma época, Leonardo da Vinci (1452-1519) describió que la rama contraria del nervio vago izquierdo (conocido después por nervio laríngeo recurrente) gira y ofrece al corazón algunas ramificaciones. Él tomó la idea de Galeno de que los nervios sensoriales eran de consistencia blanda y los motores de consistencia dura, por lo que Leonardo se preguntó si la rama era motora o sensitiva, y anotó así: "Observé en que forma los nervios contrarios llevan la sensación proveniente de los anillos traqueales y cuáles son los músculos que dan movimiento a los anillos para producir una voz profunda, media o aguda".

Andreas Vesalio (1514-1564) tuvo acercamientos conceptuales y experimentales en relación con la anatomía comparada, puesto que no entendía por qué sus contemporáneos calificaban al ser humano como un ser con facultades superiores en comparación con otras especies animales como perros, ovejas, caballos, monos y el ganado. Él observó claramente en sus disecciones que el ser humano no poseía ninguna estructura adicional, por lo que destacaba la similitud existente (Finger), incluso la laríngea con su respectiva inervación. Vesalio y posteriormente Thomas Willis (1621-1675) describieron la anatomía de la laringe y, al igual que Galeno, estudiaron el nervio laríngeo recurrente (Ardito et al). Fue a finales del siglo XVII que se revivió el interés en la anatomía animal comparada, pues la anatomía descriptiva no logró capturar la mente de la mayoría de los anatomistas, puesto que era estática y registraba solamente el aspecto, la textura y disposición de la partes. En forma extrema, no revelaba nada del uso o de las funciones de esas partes (Coleman). Willis dio un avance en este sentido, ya que en su obra dedicada al sistema nervioso, titulada Cerebri Anatome de 1664, presentó aportes de anatomía comparada nerviosa y describió además un caso de afasia en una persona que había sufrido un golpe en la cabeza con perdida momentánea de la conciencia (Finger). Posiblemente se trate del primer dato clínico que orientara en la ubicación cere- 
bral como centro de comando nervioso para la generación de la palabra, aunque a lo largo de la historia siempre ha existido un gran interés sobre los orígenes del lenguaje humano, en el que obviamente el nervio laríngeo juega un papel importante por la inervación motora.

En el antiguo Egipto, se cuenta que el faraón Psamtik tomó a dos niños y los llevó a vivir con padres sordomudos para descubrir cuál era la lengua natural de los seres humanos. Cuando observó más tarde a los niños, uno de ellos dijo algo que sonó como bekos, palabra Frigia correspondiente a pan. De esta observación, Psamtik concluyó que la lengua Frigia era en verdad la lengua original de los humanos. El tema continuó siendo altamente polémico y controvertido durante los siglos siguientes y se propusieron diferentes enfoques e interpretaciones. En un cierto momento, el debate se hizo tan complejo y contradictorio que en 1866 la Sociedad Linguística de París prohibió la discusión y argumentó que se trataba simplemente de un problema irresoluble (Ardila, 2006). Para ese entonces, el conocimiento que se tenía sobre los elementos relacionados con la voz estaban un tanto dispersos, hasta que Giovanni Battista Morgagni (1682-1771) y Heinrich August Wrisberg (1739-1808) se dedicaron a describir anatómicamente el aparato fonador humano (Entralgo), y se empezó a dar una vasta articulación conceptual integradora entre el nervio laríngeo recurrente y la laringe.

Con el paso del tiempo, los aportes de la anatomía comparada parecieron estancarse, pero un nuevo hallazgo se dio en 1835 cuando Georg Büchner, médico y escritor, dedicado a la anatomía (especialmente a la neuroanatomía), sustentó su tesis de doctorado acerca de lo nervios craneales en el barbo de río (Cyprinus barbus) e hizo énfasis en la necesidad de explicar la conformación cerebral humana a partir de la conformación anatómica de animales de una escala filogenética más temprana, como los peces y los anfibios (Pflüger \& Ayan, 2005). Posteriormente, Pierre Paul Broca (1824-1880), en el año de 1868, visitó Norwich para comentar sus hallazgos a la Sociedad Británica para el Avance de la Ciencia sobre cuatro causas diferentes para las afecciones del habla. Una de ellas era debida a un problema mecánico en el aparato fonador, como por ejemplo un daño en sus nervios periféricos (Finger; García Porrero \& Hurle). Broca describió dos pacientes que habían perdido la habilidad de hablar luego de una lesión de la parte posterior del giro frontal inferior; sin embargo, otros trabajos muestran que existen otras regiones que también juegan papel en la articulación del lenguaje (Dronkers et al., 2007). Con el advenimiento de la teoría de la evolución en el siglo XIX algunos anatomistas, como Karl Gegenbaur (1826-1903), Thomas Huxley (1825-1895) y Ernst Haeckel (1834-1919), comenzaron a estructurar, dentro del estilo arquitectónico de la descripción anatómica heredada de Vesalio, una idea evolucionista entre las diversas especies, disciplina que tomó el nombre de anatomía comparada evolucionista (Entralgo). Frank Howard Lahey (1880-1953), hacia 1923, en la ejecución de la cirugía de la glándula tiroides, enfatizó la importancia del nervio laríngeo recurrente y desarrolló una técnica quirúrgica estándar para su identificación y exposición (Ardito et al.). Este nervio tomó mayor importancia en la Medicina humana desde la década de los años cincuenta (1950) (Pascual-Font et al., 2006), debido a las complicaciones quirúrgicas que ocurrían con la extracción de la glándula tiroides como tratamiento para las enfermedades neoplásicas o endocrinas, así como en las cirugías de cuello (Entralgo; Pascual- Font et al.). Este riesgo quirúrgico se debe a la intima relación que posee el nervio con la arteria tiroidea inferior, en especial del lado derecho, haciendo probable el seccionamiento del nervio durante el acto quirúrgico (Ardito et al.; Shindo et al., 2005), o también por complicaciones postoperatorias debidas a contusión y compresión por sangre acumulada alrededor de una porción del nervio. En diversas patologías y complicaciones quirúrgicas y/o postquirúrgicas se han observado alteraciones tanto en la fonación (disfonía y afonía) como en la respiración (disnea), debidas a un daño ocasionado al nervio que impedía la conducción normal del potencial de acción. Por lo anterior, se despertó un interés clínico por la anatomía del nervio laríngeo recurrente, en especial por las relaciones que tiene con otras estructuras.

\section{CONCLUSIÓN}

Múltiples autores a lo largo de la historia han contribuido con su trabajo a un mejor entendimiento de los elementos anatómicos relacionados con la fonación y articulación de la palabra, entre ellos la laringe y el nervio laríngeo recurrente. No obstante, la ciencia sigue en procura de una mejor comprensión de aquellos mecanismos que regulan la actividad fonatoria y articulatoria, además de su control nervioso. Con cada paso alcanzado se logra una mejor aplicación en el campo quirúrgico y terapéutico. Así, los hallazgos de hoy serán la historia del futuro.

DUQUE, P. J. E.; BARCO, C. J. A.; SÁNCHEZ, R. J. D. History of comparative anatomy of the phonatory and articulatory elements. Int. J. Morphol., 32(4):1337-1340, 2014.

SUMMARY: The objective of this study was to order over time from ancient times to our time, the prevailing ideas on phonatory and articulatory organs based on functional and comparative anatomy. We reviewed various literature sources on phonatory and articulatory organs and ordered in time, in relation 
to concepts on the formation and function of these organs comparing those ideas and concepts. Historically it is shown that with the advancement of research and development of new ideas and empirical findings, came to a better understanding of phonatory and articulatory elements.

KEY WORDS: History; Phonation; Articulation; Anatomy.

\section{REFERENCIAS BIBLIOGRÁFICAS}

Aquino, S. L.; Duncan, G. R. \& Hayman, L. A. Nerves of the Thorax: Atlas of Normal and Pathologic Findings. Radiographics, 21(5):1275-81, 2001.

Ardila, A. The origins of language: an analysis from the aphasia perspective. Rev. Neurol., 43(11):690-8, 2006.

Ardito, G.; Revelli, L.; D’Alatri, L.; Lerro, V.; Guidi, M. L. \& Ardito, F. Revisited anatomy of the recurrent laryngeal nerves. Am. J. Surg., 187(2):249-53, 2004.

Baldocini, M.; Ruíz Roque, I.; Baetti, D.; Ibarzabal, J.; Quintero, D. A.; Ruíz Román, A. \& Nuñez, J. D. Relación entre el nervio laríngeo recurrente y la arteria tiroidea inferior: estudio en fetos. Rev. Arg. Anat. Online, 2(3):80-4, 2011.

Coleman, W. La biología en el siglo XIX: problemas de forma, función y transformación. México D. F., Fondo de Cultura Económica, 2002.

Dronkers, N. F.; Plaisant, O.; Iba-Zizen, M.T.; Cabanis, E.A. PPaul Broca's historic cases: high resolution MR imaging of the brains of Leborgne and Lelong. Brain, 130(Pt. 5):1432-41, 2007.

Embick, D.; Marantz, A.; Miyashita, Y.; O’Neil, W.; Sakai, K.L. A syntactic specialization for Broca's area. Proc. Natl. Acad. Sci. U. S. A., 97(11):6150-4, 2000.

Entralgo, P. L. Historia Universal de la Medicina. Barcelona, Salvat, 1982.

Finger, S. Origins of Neuroscience: A History of Explorations into Brain Function. New York, Oxford University Press, 1994.

García Ballester, L. Los orígenes del saber anatómico occidental. Med. Hist., 25(2):7-16, 1973.

Gross, C. G. Brain, vision, memory: Tales in the history of neuroscience. Cambridge (Mass), MIT Press, 1999.

López Piñero, J. M. Herófilo de Calcedonia y Erasístrato de Ceos (Siglo III a.C). Mente Cereb., 26:9-11, 2007.

Lyons, A. S. \& Petrucelli, R. J. Medicine, An Ilustrated History. New York, Harry N. Abrams, 1978.
Meng, H.; Murakami, G.; Suzuki, D. \& Miyamoto, S. Anatomical variations in stylopharyngeus muscle insertions suggest interindividual and left/right differences in pharyngeal clearance function of elderly patients: a cadaveric study. Dysphagia, 23(3):251-7, 2008.

Pascual-Font, A.; Merchán, A.; Maranillo, E.; Brillas, A.; Sañudo, J. R. \& Valderrama-Canales, F. J. Morfometría de los nervios laríngeos recurrentes de la rata. Acta Otorrinolaringol. Esp., 57(10):435-40, 2006.

Pérez Ibáñez, M. J. Galli Vocant Istvm Morbvm Morbvm Eius Cvivs Est. Otra designación para el ‘mal francés'. Asclepio. Rev. Hist. Med. Cienc., 60(1):267-80, 2008.

Plfüger, H. J. \& Ayan, S. J. Georg Büchner y la anatomía cerebral comparada. Mente Cereb., 10:72-6, 2005.

Prives, M.; Lisenkov, N. \& Bushkovich, V. Anatomía humana. Moscú, Editorial MIR, 1984.

Repici, L. The epiglottis in antiquity in medicine and philosophy. Hist. Philos. Life Sci., 12(1):67-104, 1990.

Sánchez Guisande, G. Historia de la Medicina. Buenos Aires, Atlántida, 1945.

Shindo, M. L.; Wu, J. C. \& Park, E. E. Surgical anatomy of the recurrent laryngeal nerve revisited. Otolaryngol. Head Neck Surg., 133(4):514-9, 2005.

Simonyan, K. \& Horwitz, B. Laryngeal motor cortex and control of speech in humans. Neuroscientist, 17(2):197-208, 2011.

Singh, R. \& Husain, A. M. Neurophysiologic intraoperative monitoring of the glossopharyngeal and vagus nerves. J. Clin. Neurophysiol., 28(6):582-6, 2011.

Velásquez B., M. A. La cara oculta de la neuroanatomía. Neurocuba, 2008:36-42, 2008.

von Staden, H. The discovery of the body: human dissection and its cultural contexts in ancient Greece. Yale. J. Biol. Med., 65(3):223-41, 1992.

Dirección para Correspondencia:

Jorge Eduardo Duque Parra

Departamento de Ciencias Básicas

Programa de Medicina

Facultad de Ciencias para la Salud

Universidad de Caldas

Manizales

COLOMBIA

Email: jorge.duque_p@ucaldas.edu.co

Recibido : 01-07-2013

Aceptado: 11-07-2014 